\title{
Osteocondritis disecante de rodilla en paciente pediátrico, tratado con mosaicoplastía via artroscópica
}

\section{Reporte de un caso}

\author{
Aguilar-Gómez Julio César*, Ortiz-Díaz Luis Gabrie***, Cervantes-Gudiño Jorge Enrique****
}

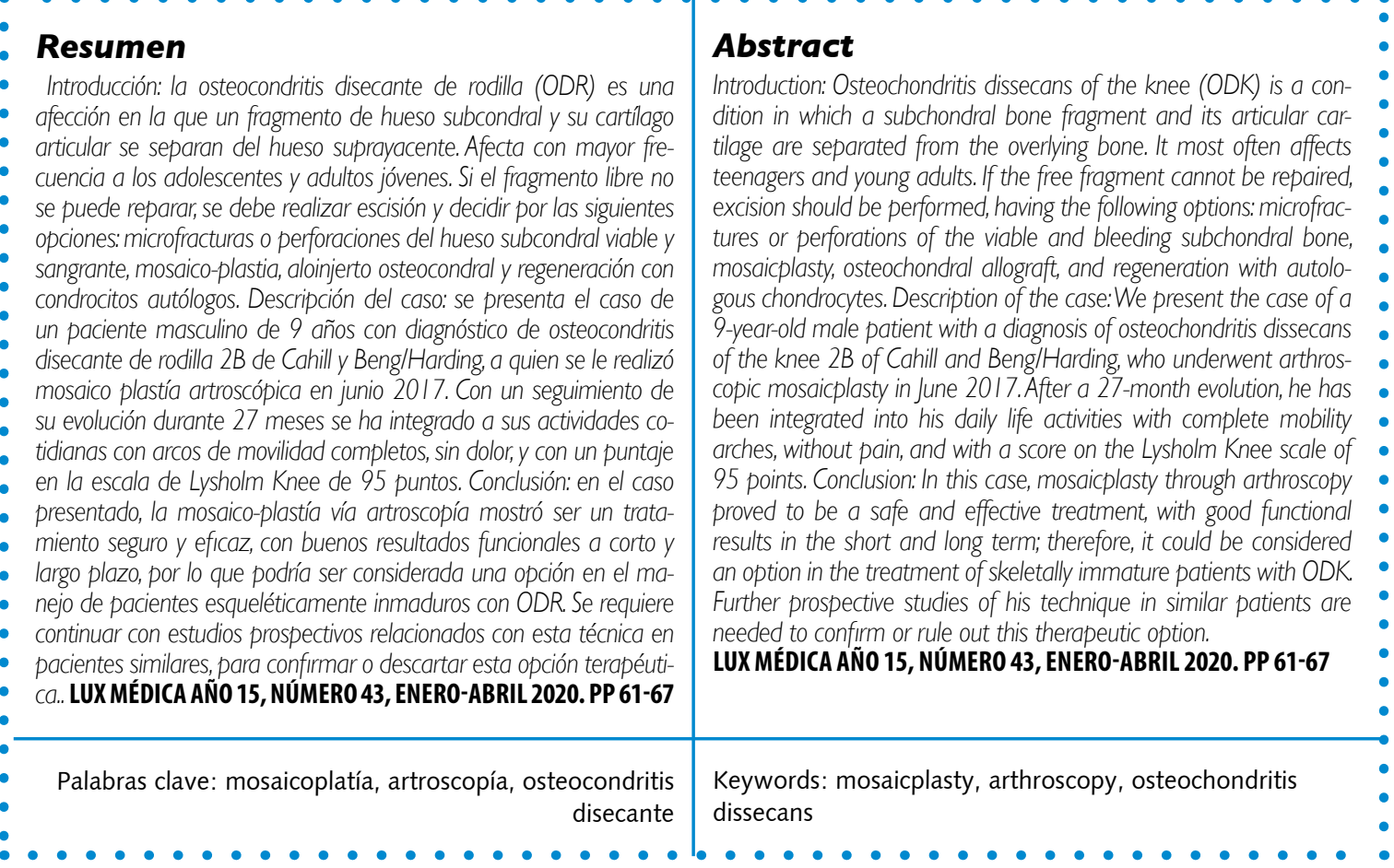

* Residente de 4to año de Traumatología y Ortopedia. Centenario Hospital Miguel Hidalgo. Aguascalientes., Ags. México

** Médico Adscrito al servicio de Traumatología y Ortopedia. Profesor titular de la especialidad. Centenario Hospital Miguel Hidalgo. Aguascalientes. Ags. México

*** Médico Adscrito al servicio de Traumatología y Ortopedia. Departamento de Ortopedia Pediátrica. Centenario Hospital Miguel Hidalgo. Aguascalientes, Ags. México

Fecha de recibido: 21 de octubre 2019

Fecha de aceptación: 30 de noviembre 2019

Correspondencia: Dr. Jorge Enrique Cervantes Gudiño. Departamento de Ortopedia y Traumatologia del Centenario Hospital Miguel Hidalgo. Av. Gómez Morín S/N. Col. La Estación, Alameda, C.P. 20259.Aguascalientes, Ags., México. Telefono 014499946720 Correo electróncio ortopedcervantesgudi@hotmail.com 


\section{Introducción}

La osteocondritis disecante de rodilla (ODR) es una afección en la que un fragmento de hueso subcondral y su cartílago articular se separan del hueso suprayacente. Afecta con mayor frecuencia a los adolescentes y adultos jóvenes, ${ }^{1}$ con una incidencia del 0.05 al $0.08 \%$ en la población general. Con respecto a su etiología, se barajan diversas posibilidades, entre las que destacan los trastornos del crecimiento, las anomalías epifisarias, los desequilibrios endocrinológicos, la predisposición familiar, los traumatismos y la necrosis avascular. Se localiza clásicamente en la porción externa del cóndilo femoral interno, otras localizaciones menos frecuentes incluyen el cóndilo externo y la tróclea. ${ }^{2}$ Comúnmente, se manifiesta con dolor, pero puede presentarse con bloqueo articular e inflamación. ${ }^{3}$ Para su diagnóstico se emplea la radiografía simple y la resonancia magnética. Su tratamiento depende de la estabilidad del fragmento y el estado del cartílago suprayacente. De forma inicial se prefiere el tratamiento conservador. Tras un periodo de 3 a 6 meses se justifica el tratamiento quirúrgico. En el caso de defectos condrales pequeños, la excéresis vía artroscópica es suficiente y en defectos grandes una alternativa es el empleo de aloinjertos osteocondrales. Las lesiones de mayor extensión localizadas en zona de carga del cóndilo femoral interno pueden resultar en artrosis. $^{4}$

La importancia del reconocimiento precoz de la ODR radica en que puede ser fácilmente confundida con otras alteraciones de la superficie articular, tales como fracturas osteocondrales, osteonecrosis, centro de osificación accesorios, osteocondrosis y displasia epifisaria. ${ }^{5}$

Existe una amplia gama de tratamientos para las lesiones inestables, si el fragmento es viable con la superficie articular se realiza vía artroscópica o abierta el curetaje del lecho óseo, la reducción y la fijación del fragmento. Si el fragmento libre no se puede reparar, se debe realizar escisión y decidir por las siguientes opciones: microfracturas o perforaciones del hueso subcondral viable y sangrante, mosaicoplastía, aloinjerto osteocondral y regeneración con condrocitos autólogos. De estos últimos, en el paciente esqueléticamente inmaduro y en menores de $2 \mathrm{~cm}^{2}$ se ha establecido como tratamiento estándar las microfracturas por la vía artroscópica. La mosaicoplastía está reservada para pacientes con alta demanda y defectos comúnmente mayor de $2 \mathrm{cms}^{2}$, reportándose en la literatura casos aplicados a pacientes esqueléticamente maduros (cierre de fisis), con excelente resultado y presentando un menor tiempo de inicio de carga y reintegración a las actividades deportivas. 


\section{Presentación del caso clínico}

Se presenta el caso de un paciente masculino de 9 años. Inició el padecimiento actual en febrero 2017, al sufrir una caída de su propia altura con contusión directa en la rodilla izquierda en flexión, recibió manejo antiinflamatorio con mejoría parcial; seis semanas después, vuelvió a sufrir otra caída de su propia altura con el mismo mecanismo de lesión. Acudió a un hospital de segundo nivel donde se feruliza durante una semana; sin embargo, persistía con limitación funcional y dolor, por lo que se tomó un estudio radiográfico y se remitió a una institución de tercer nivel a valoración, donde el servicio de oncología descartó un proceso tumoral, por lo que se derivó al servicio de Ortopedia.

Dentro de la exploración física, destaca la rodilla izquierda con un aumento de volumen de $1.5 \mathrm{~cm}$ en comparación con la contralateral, dolor a la palpación a nivel del cóndilo medial y evidente bloqueo articular, logrando una flexión máxima de 80 grados.

Se valora la radiografía anteroposterior y lateral de la rodilla izquierda, donde se observó en el cóndilo medial izquierdo un margen óseo esclerótico de aproximadamente $4 \mathrm{~mm}$, bien delimitado. Se le realizó una resonancia magnética de rodilla donde se observó un fragmento osteocondral contenido en superficie articular.
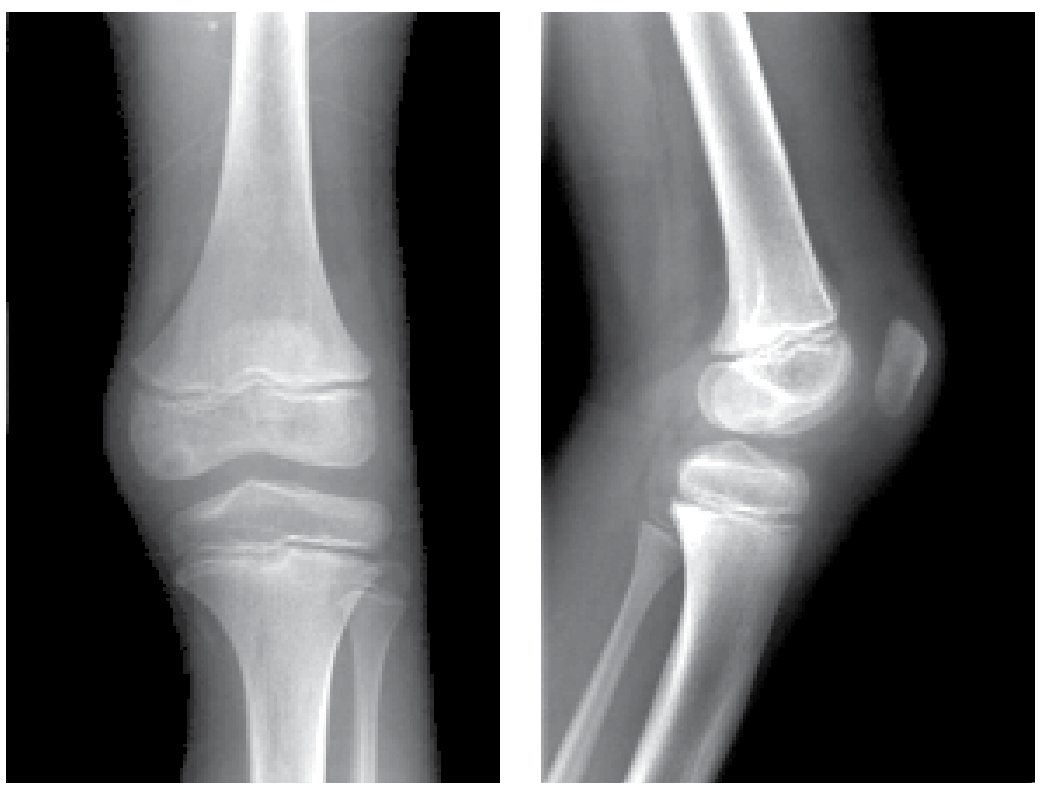

Fig 1. Radiografias anteroposterior y lateral de rodilla izquierda donde se aprecia en el condilo medial un margen óseo esclerótico de aproximadamente $4 \mathrm{~mm}$, bien delimitado. 


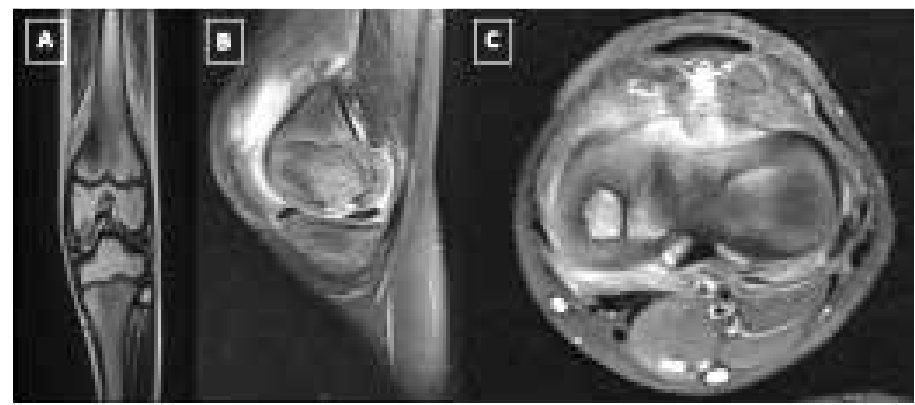

Fig 2. Imagen por resonancia magnética en T2 planos cronal (A) y sagital (B) con saturación grasa. OCD en cóndilo femoral interno de rodilla izquierda con fragmento osteocondral contenido en superficie articular, sin edema óseo adyacente. Ponderación T1 con saturación grasa con corte axial.

Se confirma el diagnóstico de ODR izquierda clasificación 2B de Cahill y Beng/ Harding, por lo que se propone mosaicoplastía artroscópica, la cual se realizó en junio 2017 con equipo para autoinjerto osteocondral (OATS) (Arthrex, USA). ${ }^{7}$

\section{Técnica quirúrgica}

Con el paciente bajo bloqueo mixto se realizó la exploración artroscópica de la rodilla mediante un portal anterolateral y anteromedial, identificando en zona de carga del cóndilo femoral medial, un defecto en sacabocado osteocondral de $8 \mathrm{~mm}$ de diámetro. Se toma el injerto osteocondral en la zona de no carga del cóndilo femoral lateral para dar cobertura en la zona de defecto mediante la técnica mencionada. Se retira el equipo de artroscopía y se da cierre a las heridas.

\section{Seguimiento}

En la consulta externa, se le difirió el apoyo del miembro afectado durante tres semanas, posteriormente se inició la carga progresiva con apoyo de muletas axilares y a rehabilitación muscular durante las siguientes cuatro semanas. Al cumplir las ocho semanas el paciente deambulaba de forma independiente y sin limitación funcional. A las diez semanas de postoperado se integró a las actividades deportivas cotidianas escolares. Actualmente, tras un seguimiento de 27 meses, se refiere asintomático; realiza actividades cotidianas con absoluta normalidad y presenta los arcos de movilidad completos, libre de dolor. Se refiere clínicamente satisfecho con un puntaje en la escala de Lysholm Knee de 95 puntos.

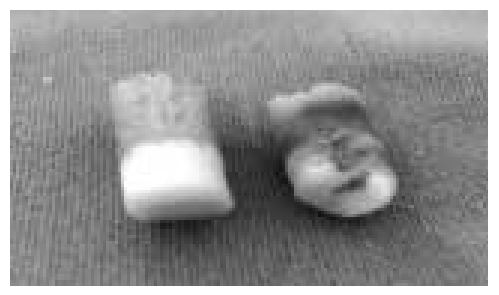

Fig 3. Fotografía de injertos tomados en el transquirúrgico. En la imagen izquierda tejido condral y subcondral sano. En la derecha pieza tomada de sitio de lesión en el cóndilo medial de rodilla izquierda, 


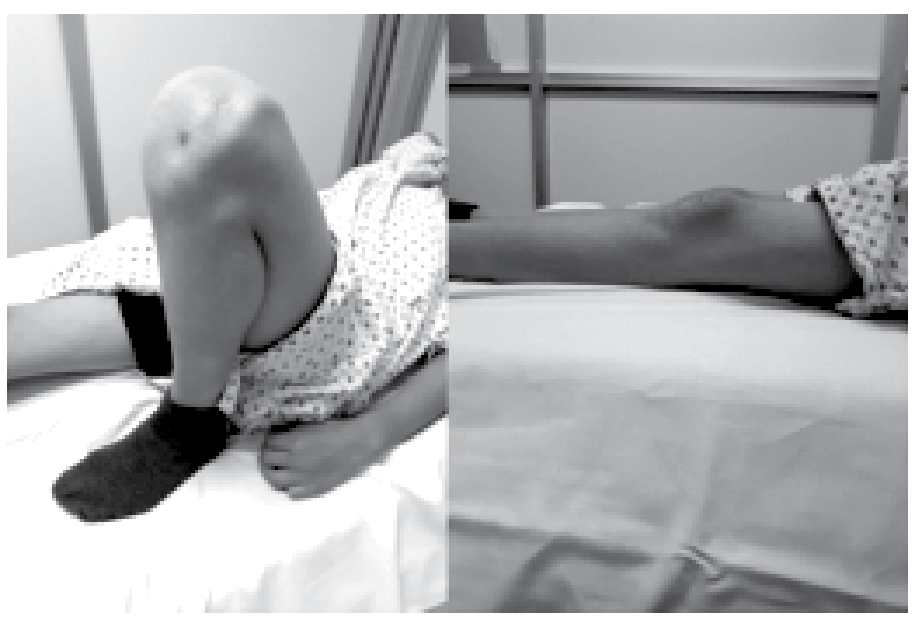

Figura 4. Exploración de arcos de movilidad, 27 meses de post-operado.

\section{Tabla I}

\section{Score de Lyshom}

\begin{tabular}{|ll|}
\hline ¿Renguea? & ¿Presenta inflamación en su rodilla? \\
\hline No (5) & No (10) \\
\hline Periodicamente (3) & Con actividad severa (6) \\
\hline Constantemente (0) & Con actividad habitual (2) \\
\hline ¿Utiliza soporte para caminar? & ¿Puede subir escaleras? \\
\hline No (5) & Sin problemas (10) \\
\hline Bastón o muleta (2) & Empeoró ligeramente (6) \\
\hline No puede apoyar Ml(0) & Un escalón a la vez (2) \\
\hline ¿Presenta inestabilidad? & Imposible (0) \\
\hline No. Nunca (25) & \\
\hline Inconstante y con ligero ejercicio (20) \\
\hline Marcado durante actividad severa (15) \\
\hline Marcado durante y después de caminar 10 km (10) \\
\hline Marcado durante y después de caminar menos de 2 km (5) \\
\hline
\end{tabular}

Este estudio reúne un puntaje máximo total de 100 pts

\section{Discusión}

Se ha demostrado que la incidencia de ODR en pacientes de 6 a 19 años fue de 9.5 por 100,000, con relación 3:1 entre pacientes masculinos y femeninos respectivamente. ${ }^{11}$

El tratamiento de la ODR del paciente esqueléticamente inmaduro depende fundamentalmente de la estabilidad de la lesión y del cartílago articular demostrada en la exploración clínica, en las pruebas de imagen y en la artroscopía. Sin duda, la mayor utilidad del uso de la artroscopía en pacientes pediátricos es la evaluación de la articulación de la rodilla. Al evaluar trabajos multicéntricos prospectivos de 413 pacientes que compararon cuatro técnicas para el tratamiento de las lesiones osteocondrales de la rodilla (perforaciones de Pridie, artroplastía por ablación, micro- 
fracturas y mosaicoplastía), se observa que la mosaico plastía obtuvo mejor resultado clínico que otras técnicas, especialmente en un seguimiento de 3, 4 y 5 años. $^{6}$ Sin embargo, este tipo de trabajos, fueron enfocados en la población adulta.

En el caso clínico presentado, paciente de la primera década de la vida, el cual cursa con un cuadro clinico clásico de una ODR, confirmado mediante estudios de gabinete estipulados. ${ }^{8}$ Se reporta el hallazgo de una lesión osteocondral de $8 \mathrm{~mm}$ de grosor y $16 \mathrm{~mm}$ de extensión, la cual es por debajo de la indicación clásica de mosaicoplastia $\left(2 \mathrm{cms}^{2}\right) .^{9} \quad$ El estándar de tratamiento para este paciente, sería realizar microfracturas en la zona del defecto osteocondral, por la via artroscópica, lo cual supone una recuperación más lenta con un incio del apoyo de la extremidad afectada tardío, comparada con la técnica de mosaicoplastía.

En nuestro hospital, no hay antecedentes del empleo de esta técnica en un paciente con características similares al de nuestro caso, tampoco se encontraron reportes en la literatura de la aplicación de esta técnica en pacientes esqueléticamente inmaduros. Se realizó una búsqueda en la plataforma PubMed introduciendo las palabras: <dissecting > <osteocondritis> $<$ knee $>$ <children > y esta arrojó sólo cuatro resultados, los cuales corresponden a literatura reportada hace más de 30 años y ninguno habla de las técnicas novedosas actuales diagnóstico-terapéutica, entre ellas, la artroscopía. No obstante, debido a que nos encontramos ante un paciente de 9 años, que es altamente demandante y con una lesión de dimensiones limítrofes, optamos por realizar una mosaicoplastia artroscópica con técnica descrita. ${ }^{7}$

La principal razón por la que no se efectua esta técnica en pacientes con las características como el nuestro, es por la potencial morbilidad que pudiera desencadenar la zona donadora de injerto en pacientes esqueléticamente inmaduros. No obstante, lo anterior no está bien fundamentado en la literatura médica publicada. La técnica de microfracturas tiene como finalidad permitir el acceso de precursores de cartílago desde la médula ósea subyacente a la superficie articular, lo que estimula una respuesta curativa. Gudas y cols, realizaron un estudio prospectivo alteatorizado donde compararon la técnica de microfracturas y la técnica OAT (mosaicoplastía) en la población esqueléticamente madura, con un seguimiento de cuatro años. Encontraron excelentes resultados en el $83 \%$ de los pacientes manejados con OAT y $63 \%$ en el grupo de tratados con microfracturas. ${ }^{12}$ La técnica de mosaicoplastía permite una restauración más fisiológica de la unidad osteocondral. Implica la cosecha e implantación de tapones osteocondrales autólogos en una sola cirugía y la morbilidad de este procedimiento se ve reducida cuando se realiza por vía artroscópica. ${ }^{13}$

Miniaci y Tytherleigh Strong, sugirieron usar la técnica de mosaicoplastía para reparar lesiones principalmente inestables por ODR. Recogieron injertos osteocondrales de $4.5 \mathrm{~mm}$ de los bordes de la tróclea que fueron insertados a través de la lesión osteocondral a una población mixta de 20 pacientes esqueléticamente maduros (entre 17 y 23 años). Seis meses después de la cirugía, todos los defectos cartilaginosos se encontraron completamente cicatrizados en la resonancia magnética, y a los 18 meses, los pacientes se encontraron completamente integrados a sus actividades deportivas cotidianas sin limitaciones. ${ }^{14}$

Al paciente que estamos reportando, se le realizó el procedimiento quirúrgico sin complicaciones y se le ofreció un manejo postoperatorio idéntico al establecido para un adulto, con apoyo diferido durante tres semanas, esperando la osteointegración del injerto autólogo, y un pronto regreso a las actividades deportivas cotidianas, debido a que con la técnica de microfracturas, la recuperación sería más insidiosa y con una evolución clínica. ${ }^{10}$ 


\section{Conclusiones}

En el caso presentado, la mosaicoplastía vía artroscopía resultó en un tratamiento seguro y eficaz, con buenos resultados funcionales a corto y largo plazo, por lo que podría ser considerada una excelente opción en el manejo de pacientes esqueléticamente inmaduros con ODR. Se requiere continuar con estudios prospectivos relacionados con esta técnica en pacientes similares para confirmar o descartar esta opción terapéutica.

\section{Bibliografía}

1. Alvarez A, Garcia. Mosaicplasty. Rev Arch Med Camagüey 2017;21(1): 902-912.

2. Courtin $F$, Chotel $E$, Servein $P$, Neyret, $S$, et al. Osteochondritis Dissecans of the Knee. Rev Esp Cir Ortop Traumatol 2002; 46:428-35.

3. Jeffrey I, Hooman N, Kevin G, Shea, John C, et al. The Demographics and Epidemiology of Osteochondritis Dissecans of the Knee in Children And Adolescents. Am J sports Med 2014;42: 320-326.

4. Grimm NL, Tisano B, Carey JL. Three osteochondritis dissecans lesions in one knee: a case report. Clin Orthop Relat Res 2013;471(4):1186-1190.

5. Canallas A, Plantalamor L, Cañellas A, Juanico A. The Osteocondritis Dissecans (Associated Marks in Adult Age). Laboratorio de Paleopatologia del Museu de Menorca. 2013;567-75.

6. Vannini F, Battaglia M, Buda R, Cavallo M, Giannini S. "One step" treatment of the juvenile osteochondritis dissecans in the knee: clinical results and T2 mapping characterization. Orthop Clin N Am. 2012; 43 (2): 237-244.

7. Arthrex; Técnica [1]OATS $®$ para aloinjerto. Disponible en: https://www.arthrex.com/es/etiquetas/ mosaicoplastia-con-aloinjerto

8. Edmonds EW, Polousky J. A review of knowledge in osteochondritis dissecans: 123 years of minimal evolution from Konig to the ROCK study group. Clin Orthop Relat Res 2013;471(4):1118-26.
9. Astur D, Arliani G, Binz M, et al. Autologus Ostechondral Transplantation for Treating Patellar Chondral Injuries. J Bone Joint Surg Am. 2014;96:816-23

10. Kocher $M$, Tucker $R$, Ganley $T$, et al. Management of Osteochondritis Dissecans of the Knee. Am J Sports Med. Julio de 2006; 34 (7): 1181-91

11. Bruns J, Werner M, Habermann C. Osteochondritis Dissecans: Etiology. Pathology, and Imagin with a Special Focus on the Knee Joint. Cartilage. 2018 Oct; 9 (4):346-362.

12. Gudas R, Simonaityte R, Cekanauskas E, Tamosiunas $R$ A prospective, randomized clinical study of osteochondral autologous transplantation versus microfracture for the treatment of osteochondritis dissecans in the knee joint in children. J Pediatr Orthop 2009; 29:741-748.

13. Marcacci $M$, Filardo $G$, Kon E Treatment of cartilage lesions: what works and why? Injury 2013;44:1115.

14. Miniaci A, Tytherleigh-Strong G Fixation of unstable osteochondritis dissecans lesions of the knee using arthroscopic autogenous osteochondral grafting (mosaicplasty). Arthroscopy 2007 23:845-851.

15. Filardo $G$, et al. Treatment of unstable knee osteochondritis dissecans in the young adult: results and limitations of surgical strategies-The advantages of allografts to address an osteochondral challenge. Knee Surg Sports Traumatol Arthrosc. 2019; 27(6): 1726-1738. 